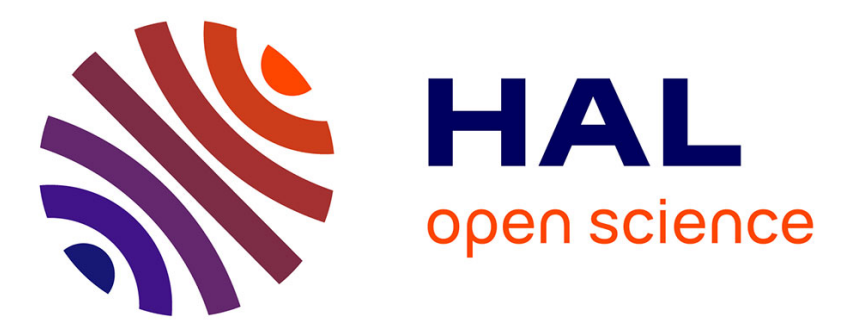

\title{
Nonlinear event-triggered tracking control of a mobile robot: design, analysis and experimental results
}

Romain Postoyan, Marcos Cesar Bragagnolo, Ernest Galbrun, Jamal Daafouz, Dragan Nesic, Eugênio Castelan

\section{- To cite this version:}

Romain Postoyan, Marcos Cesar Bragagnolo, Ernest Galbrun, Jamal Daafouz, Dragan Nesic, et al.. Nonlinear event-triggered tracking control of a mobile robot: design, analysis and experimental results. 9th IFAC Symposium on Nonlinear Control Systems, NOLCOS 2013, Sep 2013, Toulouse, France. pp.318-323. hal-00875659

\section{HAL Id: hal-00875659 https://hal.science/hal-00875659}

Submitted on 22 Oct 2013

HAL is a multi-disciplinary open access archive for the deposit and dissemination of scientific research documents, whether they are published or not. The documents may come from teaching and research institutions in France or abroad, or from public or private research centers.
L'archive ouverte pluridisciplinaire HAL, est destinée au dépôt et à la diffusion de documents scientifiques de niveau recherche, publiés ou non, émanant des établissements d'enseignement et de recherche français ou étrangers, des laboratoires publics ou privés. 


\title{
Nonlinear event-triggered tracking control of a mobile robot: design, analysis and experimental results
}

\author{
R. Postoyan* M.C. Bragagnolo* E. Galbrun* J. Daafouz * \\ D. Nešićc* E.B. Castelan ${ }^{* * *}$ \\ * Université de Lorraine, CRAN, UMR 7039 and CNRS, CRAN, UMR \\ 7039, France (e-mails: \{romain.postoyan, marcos-cesar.bragagnolo, \\ ernest.galbrun, jamal.daafouz\}@univ-lorraine.fr). \\ ** Department of Electrical and Electronic Engineering, the University \\ of Melbourne, Parkville, VIC 3010, Australia (e-mail: \\ dnesic@unimelb.edu.au) \\ *** Grupo de Controle de Sistemas Mecatronicos - CSM Depto. de \\ Automação e Sistemas - DAS Universidade Federal de Santa Catarina \\ - UFSC Florianópolis, Brasil (e-mail: eugenio@das.ufsc.br)
}

\begin{abstract}
We consider the scenario where a controller communicates with a mobile robot via a network. Our objective is to design the control law such that the robot tracks a given reference trajectory while reducing the usage of the communication channel. For that purpose, we design a nonlinear event-triggered feedback law. We follow an emulation-like approach in the sense that we first synthesize the controller while ignoring the communication constraints and we then derive an appropriate triggering condition. We prove that the states of the robot's model practically converge towards the states of the reference system which generates the desired trajectory to be tracked, using an invariance principle for hybrid systems. The existence of a dwell-time between any two transmissions is discussed. We have implemented the proposed strategy on a benchmark where the controller is located on a remote computer which communicates with the mobile robot via a IEEE $802.11 \mathrm{~g}$ wireless network. The proposed event-triggering strategy is shown to significantly reduce the need for communication compared to a classical time-triggered setup while ensuring similar, if not better, tracking performances.
\end{abstract}

\section{INTRODUCTION}

Event-triggered control is currently attracting a lot of attention because of its abilities to significantly reduce the need for communication in control systems, which is extremely useful in networked control systems. The idea is to transmit data between the controller and the plant whenever a state-dependent criterion is satisfied and not periodically as in traditional setups. In that way, the transmissions are adapted to the state of the system and they only occur when it is needed. Various techniques have been developed, see Arzén [1999], Astrom and Bernhardsson [2002], Heemels et al. [2009], Henningsson et al. [2008], Tabuada [2007] to mention a few. Most of them address the stabilization of equilibrium points, while very few controllers have been synthesized to stabilize timevarying trajectories, see e.g. Tallapragada and Chopra [2011]. It appears that tracking control induces additional difficulties as only approximate tracking can usually be ensured under communication constraints because of the time-varying component of the control law, see for more detail Postoyan et al. [2012]. As a consequence, available results on the stabilization of equilibrium points are not directly applicable in this context.

In this paper, we investigate the event-triggered tracking control of a mobile robot whose dynamics is described by a nonholonomic system. We follow an emulation-like approach (see e.g. Tabuada [2007], Nešić and Teel [2004]). Thus, we first design the controller while ignoring the communication constraints and we derive the triggering strategy afterwards. We have selected the state-feedback controller of Jiang and Nijmeijer [1997] among others, because the law is continuous and an explicit Lyapunov function is provided. The continuity of the feedback law helps to guarantee the existence of a minimum amount of time between any two transmissions, under some conditions on the reference trajectories. This point is essential in practice as the hardware cannot transmit infinitely fast. The Lyapunov function is used to design the eventtriggering condition. It has to be noted that it is a weak Lyapunov function in the sense that it does not strictly decrease along the solutions of the closed-loop system (in the absence of communication constraints), see Jiang and Nijmeijer [1997]. This is an important difference with existing Lyapunov-based event-triggered controllers, which rely on strong Lyapunov functions (see Tabuada [2007], Postoyan et al. [2011], Seuret and Prieur [2011] and the references therein).

We model the problem using the hybrid formalism of Goebel et al. [2012], like in Donkers and Heemels [2012], Forni et al. [2010], Postoyan et al. [2011], Seuret and Prieur [2011]. The event-triggering condition is inspired 
by the technique in Anta and Tabuada [2012], Forni et al. [2010] where the Lyapunov function is forced to decrease at a certain rate, except that we exclude a region of the state-space, which we call a dead-zone, to allow the existence of dwell-times. We prove that the solutions of the robot's model converge to a neighborhood of the reference trajectory under some conditions on these references. This neighborhood depends on a tuneable parameter $\varepsilon$ which defines the dead-zone of the triggering condition. The parameter $\varepsilon$ can be used to adjust the accuracy of the tracking error at the price of more transmissions. The analysis relies on an invariance principle for hybrid systems developed in Sanfelice et al. [2007]. The existence of a dwell-time is discussed and sufficient conditions are given to ensure it. Finally, the designed controller has been implemented on a benchmark where the controller is located on a remote controller and communicates with the robot via a IEEE $802.11 \mathrm{~g}$ wireless network. The obtained results show that we can significantly reduce the amount of transmission compared to periodic sampling while ensuring similar, if not better, performances. Notice that we do not rely on a local linear approximation of the plant's dynamics as in other studies where experimental tests have been lead (see Heemels et al. [1999], Lehmann and Lunze [2011], Henningsson and Cervin [2009], TéllezGuzmán et al. [2012]) but on a global nonlinear model.

The paper is organized as follows. In Section 2, the controller of Jiang and Nijmeijer [1997] is recalled and the hybrid model is introduced. The event-triggering condition is designed in Section 3 where the analytical results are presented. Section 4 shows the experimental results. The proofs have been omitted for space reasons.

Notation. Let $\mathbb{R}=(-\infty, \infty), \mathbb{R}_{\geq 0}=[0, \infty), \mathbb{R}_{>0}=$ $(0, \infty), \mathbb{Z}_{\geq 0}=\{0,1,2, \ldots\}, \mathbb{Z}_{>0}=\{1,2, \ldots\}$. For $(x, y) \in$ $\mathbb{R}^{n+m}$, the notation $(x, y)$ stands for $\left[x^{\mathrm{T}}, y^{\mathrm{T}}\right]^{\mathrm{T}}$. For a piecewise continuous function $f: \mathbb{R} \rightarrow \mathbb{R}^{n}, f\left(t^{+}\right)$stands for $\lim _{s \rightarrow t, s>t} f(s)$ for $t \in \mathbb{R}$. We recall that the function sinc is defined from $\mathbb{R}$ to $\mathbb{R}$ by $\operatorname{sinc}(x)=\frac{\sin x}{x}$ when $x \neq 0$, and $\operatorname{sinc}(0)=1$, and it is continuously differentiable with the derivative $\operatorname{sinc}^{\prime}(x)=\frac{x \cos x-\sin x}{x^{2}}$ when $x \neq 0$, and $\operatorname{sinc}^{\prime}(0)=0$.

\section{SYSTEM MODEL}

\subsection{Jiang and Nijmeijer's controller}

We consider a mobile robot for which the dynamics are defined by the following nonholonomic system

$$
\dot{x}=v \cos (\theta), \dot{y}=v \sin (\theta), \dot{\theta}=w,
$$

where $(x, y)$ are the Cartesian coordinates and $\theta$ is the angle between the heading direction and the $x$-axis, $(v, w)$ denotes the control input vector, see Figure 1. Our objective is to make the system (1) track a given trajectory $\left(x_{r}, y_{r}, \theta_{r}\right)$. We restrict our attention to the case where $\left(x_{r}, y_{r}, \theta_{r}\right)$ satisfies the following dynamical equations

$$
\dot{x}_{r}=v_{r} \cos \left(\theta_{r}\right), \dot{y}_{r}=v_{r} \sin \left(\theta_{r}\right), \dot{\theta}_{r}=w_{r},
$$

where $v_{r}$ and $w_{r}$ are the feedforward inputs to system (2). In practice, the term $w_{r}$ is obtained by differentiating $\theta_{r}$. The term $v_{r}$ is deduced by using that $v_{r}^{2}=\left(\dot{x}_{r}\right)^{2}+\left(\dot{y}_{r}\right)^{2}$ and its sign is obtained by investigating the first equations

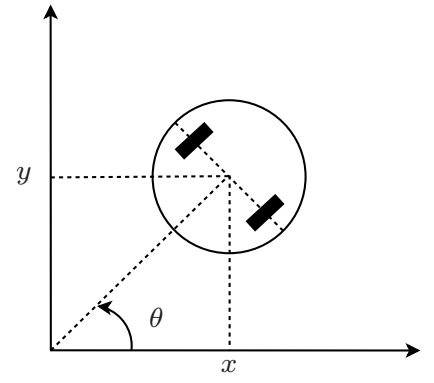

Fig. 1. Mobile robot.

of (2) for the given trajectory $\left(x_{r}, y_{r}, \theta_{r}\right)$. We refer to (2) as the reference system. We use the following error coordinates (see Kanayama et al. [1990])

$$
\left(\begin{array}{l}
x_{e} \\
y_{e} \\
\theta_{e}
\end{array}\right)=\left(\begin{array}{ccc}
\cos (\theta) & \sin (\theta) & 0 \\
-\sin (\theta) & \cos (\theta) & 0 \\
0 & 0 & 1
\end{array}\right)\left(\begin{array}{l}
x_{r}-x \\
y_{r}-y \\
\theta_{r}-\theta
\end{array}\right)
$$

which allow us to derive the error system

$$
\begin{aligned}
& \dot{x}_{e}=w y_{e}-v+v_{r} \cos \left(\theta_{e}\right) \\
& \dot{y}_{e}=-w x_{e}+v_{r} \sin \left(\theta_{e}\right) \\
& \dot{\theta}_{e}=w_{r}-w .
\end{aligned}
$$

It has to be noticed that the change of coordinates in (3) is invertible and that the transformation matrix and its inverse are bounded. As a consequence, if we can guarantee that $x_{e}, y_{e}, \theta_{e}$ converge to a neighborhood of the origin, then so do $x_{r}-x, y_{r}-y, \theta_{r}-\theta$. In Jiang and Nijmeijer [1997], the following controller is shown to ensure the global convergence of $x_{e}, y_{e}, \theta_{e}$ towards the origin (under some conditions on the feedforward terms $v_{r}$ and $w_{r}$ )

$$
v=v_{1}+c_{4} \bar{x}_{e}, w=w_{r}+\gamma y_{e} v_{r} \operatorname{sinc} \theta_{e}+c_{5} \gamma \theta_{e},
$$

where $c_{4}, c_{5}, \gamma>0$ are design parameters and

$$
\begin{aligned}
& \bar{x}_{e}:=x_{e}-c_{3} w y_{e} \\
& v_{1}:=v_{r} \cos \theta_{e}-c_{3} \dot{w} y_{e}+c_{3} w\left(w x_{e}-v_{r} \sin \theta_{e}\right)
\end{aligned}
$$

with $c_{3}>0$. We do not necessarily require to differentiate online $\dot{w}$ to compute the term $v_{1}$ in (6) as we can replace $\dot{w}$ by its algebraic expression by using (1), (2) and (5).

\subsection{Assumptions on the feedforward terms}

In Section 3, we will use an invariance principle developed in Sanfelice et al. [2007] to prove some stability properties hold for the event-triggered controlled system. We will need for that to work with an autonomous system which generates solutions that have a compact range (see Sanfelice et al. [2007]) which loosely speaking means that it exhibits bounded solutions. We see that the system (4) is time-varying and thus non-autonomous in general when the terms $v_{r}$ and $w_{r}$ are time-varying. The assumption below allows us to derive an autonomous system from (4) under some conditions on the feedforward terms $v_{r}$ and $w_{r}$.

Assumption 1. The signals $v_{r}$ and $w_{r}$ can be written as the output of a bounded autonomous dynamical system i.e. there exist a continuous vector field $f_{\chi}: \mathbb{R}^{n_{\chi}} \rightarrow \mathbb{R}^{n_{\chi}}$, a continuously differentiable vector field $u: \mathbb{R}^{n_{\chi}} \rightarrow \mathbb{R}^{2}$ (where $n_{\chi} \in \mathbb{Z}_{>0}$ ) and $\chi_{0} \in \mathbb{R}^{n_{\chi}}$ such that

$$
\left(v_{r}(t), w_{r}(t)\right)=u(\chi(t)) \quad \forall t \geq 0
$$

where $\chi(t)$ is the solution of $\dot{\chi}=f_{\chi}(\chi)$ with initial condition $\chi(0)=\chi_{0}$ and such that the closure of the set $\left\{z \in \mathbb{R}^{n_{\chi}}: \exists t \geq 0 \quad z=\chi(t)\right\}$ is compact. 
Assumption 1 allows us to transform the system (4) into an autonomous system with the extended state $\left(x_{e}, y_{e}, \theta_{e}, \chi\right)$ and $v_{r}, w_{r}$ being functions of $\chi$. One might argue that it suffices to introduce the time as an extra variable to derive an autonomous system. However, the solution of the system will then not be bounded because of the time variable. That is the reason why we have imposed Assumption 1. It has to be emphasized that our results apply to a class of reference trajectories (which are solutions to (2) and of the $\chi$-system of Assumption 1), rather than a single trajectory. A simple sufficient condition to verify the satisfaction of Assumption 1 is given below.

Lemma 2. If there exist $\alpha_{1}, \ldots, \alpha_{m}, \beta_{1}, \ldots, \beta_{m} \in \mathbb{R}$ with $m \in \mathbb{Z}_{>0}$ and a continuously differentiable vector field $u: \mathbb{R}^{n_{\chi}} \rightarrow \mathbb{R}^{2}$ such that $\left(v_{r}(t), w_{r}(t)\right)=u\left(\sin \left(\alpha_{1} t+\right.\right.$ $\left.\left.\left.\beta_{1}\right), \ldots, \sin \left(\alpha_{m} t+\beta_{m}\right)\right)\right)$ for all $t \geq 0$, then Assumption 1 holds.

It is important to emphasize that we do not need to explicitly build up the $\chi$-system of Assumption 1 in the remaining part of the paper but we only need to make sure that such a system does exist. Assumption 1 implies the following properties which correspond to the first conditions of Proposition 2 in Jiang and Nijmeijer [1997].

Lemma 3. Under Assumption 1, there exists an initial condition $\chi(0) \in \mathbb{R}^{n_{\chi}}$ for the $\chi$-system of Assumption 1 such that the signals $v_{r}, w_{r}, \dot{v}_{r}$ and $\dot{w}_{r}$ are bounded on $[0, \infty)$ by $M>0$.

We also make the following assumption on the feedforward terms which is satisfied by the reference trajectories studied in Section 4.

Assumption 4. One of the following conditions needs to hold. (i) The signal $w_{r}$ is not constantly equal to 0 , i.e. $\exists t \geq 0$ such that $w_{r}(t) \neq 0$. (ii) The signal $v_{r}$ is not constant, i.e. $\exists t_{1}, t_{2} \geq 0$ such that $v_{r}\left(t_{1}\right) \neq v_{r}\left(t_{2}\right)$.

These assumptions are used to ensure the convergence of the states of the robot's model (1) towards the state of the reference system (2). These are different from the conditions of Proposition 2 in Jiang and Nijmeijer [1997] where $v_{r}$ or $w_{r}$ is required not to converge to zero. We do tolerate $v_{r}$ and $w_{r}$ to converge to zero but $w_{r}$ cannot be constantly equal to zero or $v_{r}$ cannot be constant. These conditions come from the stability analyzes which are different: Barbalat's lemma is used in Jiang and Nijmeijer [1997] while invariance principles will be invoked here.

\subsection{Controller implementation $\&$ hybrid model}

We consider the scenario where the controller $(v, w)$ in (5) is digitally implemented and communicates with the robot (1) via a network, see Figure 2. We assume that the controller has access to the robot's measurements, the reference trajectory and the feedforward terms $v_{r}$ and $w_{r}$. Our objective is to reduce the usage of the network from the controller to the robot while ensuring the convergence of the robot's states towards the reference trajectory. We will design for that purpose an event-triggering condition which will decide when the robot's input needs to be updated. We write the obtained model using the formalism of Goebel et al. [2012] like in Donkers and Heemels [2012], Forni et al. [2010], Postoyan et al. [2011], Seuret and Prieur [2011]

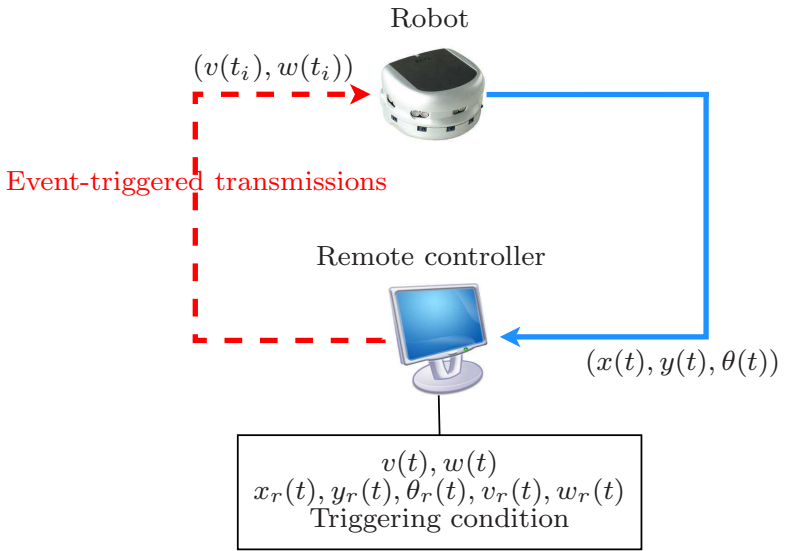

Fig. 2. Controller implementation.

$$
\begin{aligned}
& \left.\dot{x}_{e}=\left(w+e_{w}\right) y_{e}-v-e_{v}+v_{r} \cos \left(\theta_{e}\right)\right) \\
& \dot{y}_{e}=-\left(w+e_{w}\right) x_{e}+v_{r} \sin \left(\theta_{e}\right) \\
& \dot{\theta}_{e}=w_{r}-w-e_{w} \\
& \dot{e}_{w}=f_{w}(q) \\
& \dot{e}_{v}=f_{v}(q) \\
& \dot{\chi}=f_{\chi}(\chi) \\
& x_{e}^{+}=x_{e} \\
& y_{e}^{+}=y_{e} \\
& \theta_{e}^{+}=\theta_{e} \\
& e_{w}^{+}=0 \quad q \in D \\
& e_{v}^{+}=0 \\
& \left.\chi^{+}=\chi\right\}
\end{aligned}
$$

where $q:=\left(x_{e}, y_{e}, \theta_{e}, e_{w}, e_{v}, \chi\right), e_{v}:=\hat{v}-v$ and $e_{w}:=\hat{w}-$ $w$ (where $\hat{v}, \hat{w}$ are respectively the values of $v, w$ at the last jump instant), $\chi$ comes from Assumption 1 and $v, w$ are defined in (5). The sets $C$ and $D$ are closed and are defined by the triggering condition. Indeed, the set $C$ corresponds to the region of the state-space where the triggering condition is not satisfied and $D$ is defined as the region of the state-space where the triggering condition is verified. In that way, defining the triggering condition is equivalent to defining the sets $C$ and $D$. We recall that when $q \in C \cap$ $D$, the system can either jump or flow, the latter only if flowing keeps $q$ in $C$. For more detail about hybrid systems of the form of (8), see Goebel et al. [2012]. The vector fields $f_{w}$ and $f_{v}$ are respectively obtained by computing the algebraic expressions of $-\dot{w}$ and $-\dot{v}$ using (5) and (8). We use the notation $\phi=\left(\phi_{x_{e}}, \phi_{y_{e}}, \phi_{\theta_{e}}, \phi_{e_{w}}, \phi_{e_{v}}, \phi_{\chi}\right)$ to denote a solution to (8) in the following in order to avoid any confusion with the state vector $q=\left(x_{e}, y_{e}, \theta_{e}, e_{w}, e_{v}, \chi\right)$. We consider the term $v_{r}$ and $w_{r}$ in (8) as functions of $\chi$. In that way, the system (8) is autonomous. We recall the following definitions which will be used in the following.

Definition 5. (Goebel et al. [2008]). We say that a solution $\phi$ to (8) is:

- complete if its domain ${ }^{1} \operatorname{dom} \phi$ is unbounded.

- a dwell-time solution if there exists $\tau \in \mathbb{R}_{>0}$ such that for any $j \in \mathbb{Z}_{\geq 0}, t_{j+1}-t_{j} \geq \tau$ where $\operatorname{dom} \phi=\bigcup\left(\left[t_{j}, t_{j+1}\right], j\right)$.

For the sake of convenience, we denote

$$
\dot{q}=f(q) \quad q \in C, \quad q^{+}=g(q) \quad q \in D .
$$

\footnotetext{
1 The domain of $\phi$ is the subset of $\mathbb{R}_{\geq 0} \times \mathbb{Z}_{\geq 0}$ where $\phi$ is defined, see Goebel et al. [2012] for more detail.
} 
We will now design the triggering condition, i.e. the sets $C$ and $D$ in (8), such that the errors $x_{e}, y_{e}, \theta_{e}$ practically converge to the origin and such that the usage of the communication channel, i.e. the number of jumps for system (8), is minimized. The tuneable parameter will be given by the triggering condition.

\section{EVENT-TRIGGERED TRACKING CONTROL}

\subsection{Design of the triggering condition}

We follow the main steps of the proof of Proposition 2 in Jiang and Nijmeijer [1997] to build up the event-triggering condition. The Lyapunov-like candidate function below is considered in Jiang and Nijmeijer [1997]

$$
V(q)=\frac{1}{2} \bar{x}_{e}^{2}+\frac{1}{2} y_{e}^{2}+\frac{1}{2 \gamma} \theta_{e}^{2}
$$

where $\bar{x}_{e}$ is defined in (6) and $\gamma$ comes from (5). By differentiating the function $V$ along the vector fields of the system (9) on the set $C$, we obtain, by following similar lines as in Jiang and Nijmeijer [1997],

$$
\langle\nabla V(q), f(q)\rangle=-\Sigma(q)+\Lambda(q)
$$

where $\Sigma(q):=c_{3} w^{2} y_{e}^{2}+c_{4} \bar{x}_{e}^{2}+c_{5} \theta_{e}^{2}$ and $\Lambda(q):=\bar{x}_{e}\left(e_{w} y_{e}-\right.$ $\left.e_{v}-c_{3} e_{w} \rho\left(x_{e}, y_{e}, \theta_{e}, v_{r}\right) y_{e}+c_{3} w e_{w} x_{e}\right)-y_{e} e_{w} x_{e}-\frac{1}{\gamma} \theta_{e} e_{w}$, with $\rho\left(x_{e}, y_{e}, \theta_{e}, v_{r}\right):=-\gamma x_{e} v_{r} \operatorname{sinc} \theta_{e}-\left(\gamma y_{e} v_{r} \operatorname{sinc}^{\prime}\left(\theta_{e}\right)+\right.$ $\left.c_{5} \gamma\right)$. We note that we recover (41) in Jiang and Nijmeijer [1997] when $e_{v}=e_{w}=0$ (as we have in this case $\Lambda(q)=0$ ).

We use (11) to design the triggering condition. We define the flow and the jump sets as follows

$$
\begin{aligned}
C & :=\left\{q:\langle\nabla V(q), f(q)\rangle \leq-\sigma \Sigma(q) \text { or } V(q) \leq \varepsilon^{2}\right\} \\
D & :=\left\{q:\langle\nabla V(q), f(q)\rangle \geq-\sigma \Sigma(q) \text { and } V(q) \geq \varepsilon^{2}\right\}
\end{aligned}
$$

where $\sigma \in(0,1)$ and $\varepsilon>0$ are design parameters. The idea is to force $V$ to decrease with the rate $\sigma \Sigma(q)$ like in Tabuada [2007], where $\sigma \in(0,1)$. We take $\sigma \in(0,1)$ in order to allow some flow between two jumps, see e.g. Tabuada [2007] or Section III in Anta and Tabuada [2012]. In addition, we impose that condition only when $V(q) \geq \varepsilon^{2}$ as we cannot otherwise guarantee the existence of dwelltime solutions to (8) in general, as discussed in more detail in Section 3.3. It has to be noticed that $C \cup D=\mathbb{R}^{n_{q}}$.

Similar ideas are followed in Forni et al. [2010], Anta and Tabuada [2012], Postoyan et al. [2011], Seuret and Prieur [2011] to design the triggering condition. Nevertheless, none of the strategies proposed in these papers directly applies to our problem. Indeed, the triggering condition is designed based on a weak Lyapunov function $V$ (as opposed to strong Lyapunov functions) which induces nontrivial difficulties to prove stability: we need to resort to invariance principles for hybrid systems (see Sanfelice et al. [2007]) and the existence of dwell-times is a priori not ensured for every solution. We therefore have to modify the policy and to derive a different stability analysis.

\subsection{Stability results}

We are ready to state the main results of this section.

Theorem 6. Consider the system (8) and suppose Assumptions 1 and 4 are satisfied, then the following holds. (i) Any solution to (8) is complete.

(ii) For any $\Delta \geq 0$ there exists $\bar{\Delta} \geq 0$ such that for any solution $\phi$ to (8) with ${ }^{2}|\phi(0,0)| \leq \Delta$, it holds that $|\phi(t, j)| \leq \bar{\Delta}$ for all $(t, j) \in \operatorname{dom} \phi$.

(iii) Any dwell-time solution to (8) with $\phi_{e_{w}}(0,0)=$ $\phi_{e_{v}}(0,0)=0$ approaches $^{3}$ the set $\left\{q: \max \left\{\left|x_{e}\right|,\left|y_{e}\right|\right.\right.$, $\left.\left.\left|\theta_{e}\right|\right\} \leq \bar{M} \varepsilon\right\}$ with $\bar{M}=\sqrt{2} \max \{1, \sqrt{\gamma}, M+\gamma \sqrt{2} M+$ $\left.c_{5} \gamma^{\frac{3}{2}} \sqrt{2}\right\}$ where $\gamma, c_{5}$ and $M$ come from (5) and Lemma 3 .

Item (ii) of Theorem 6 ensures that the states of system (8) are bounded. Item (iii) of Theorem 6 guarantees that any dwell-time solution to (8) converges to a neighborhood of the origin whose 'size' depends on the design parameter $\varepsilon$. Therefore, by taking $\varepsilon$ small, we ensure the convergence of the states of the robot closer to the reference trajectory. Nevertheless, this may give rise to more transmissions as discussed in Section 4. The fact that the solution needs to start from zero for the $e_{v}$ and $e_{w}$ variables in item (iii) of Theorem 6 adds no conservatism. Indeed, it suffices to transmit the control input to the robot once at the beginning of the experiment and to take this time to be the initial time to guarantee it (note that the system (8) satisfies the semi-group property, see Sanfelice et al. [2007]). It has to be noticed that Theorem 6 does not ensure the existence of dwell-time solutions to (8): this is discussed in the following.

\subsection{Existence of dwell-time solutions}

First, note that the system (8) may generate Zeno solutions (i.e. solutions which constantly jump) since $g(D) \cap$ $D \neq \emptyset$. Indeed, take $q \in \mathcal{A}$ where $\mathcal{A}:=\{q: \Sigma(q)=$ 0 and $\left.V(q) \geq \varepsilon^{2}\right\}$. Then $q \in D$ and $G(q) \in D$. As a consequence, we cannot guarantee that every solution to (8) is a dwell-time solution in general. The following proposition provides sufficient conditions to ensure that there always exist dwell-time solutions to (8).

Proposition 7. Consider system (8) and suppose the following holds for a solution $\phi$ to (8).

(i) Assumptions 1 and 4 hold.

(ii) There exists $\delta>0$ such that $\left|w_{r}(t, j)\right| \geq \delta$ for all $(t, j) \in \operatorname{dom} \phi$.

(iii) The initial condition $\phi(0,0)$ is such that $\mid\left(\phi_{x_{e}}(0,0)\right.$, $\left.\phi_{y_{e}}(0,0), \phi_{\theta_{e}}(0,0)\right) \mid \leq \Delta$ where $\Delta \geq 0$ and $\gamma(M+$ $\left.c_{5}\right) \max \left\{\Delta\left(2+c_{3}\left(M+\gamma \Delta M+c_{5} \gamma\right)\right) \sqrt{\underline{\gamma}^{-1} \bar{\gamma}}, \varepsilon \sqrt{\underline{\gamma}^{-1}}\right\}$ $<\delta$, with $\underline{\gamma}=\frac{1}{2} \min \{1, \gamma\}$ and $\bar{\gamma}=\frac{1}{2} \max \{1, \gamma\}$ and where $\gamma, c_{5}, c_{3}, M$ come from (5), (6) and Lemma 3.

Then $\phi$ is a dwell-time solution.

Item (ii) of Proposition 7 means that the feedforward term $w_{r}$ has the same sign for all time and does not converge to the origin. It is satisfied in scenarios where the reference trajectory follows an ellipsoidal path or a

\footnotetext{
2 Throughout the paper, the initial condition $\phi(0,0)$ is always assumed to be such that $\phi_{\chi}(0,0)=\chi_{0}$ where $\chi_{0}$ comes from Assumption 1.

3 A solution $\phi$ to (8) approaches the set $\mathcal{A} \subset \mathbb{R}^{n}$ if for all $\epsilon>0$, there exists $(\bar{t}, \bar{j}) \in \operatorname{dom} \phi$ such that for all $(t, j)$ satisfying $(t, j) \succcurlyeq(\bar{t}, \bar{j})$, $(t, j) \in \operatorname{dom} \phi, \phi(t, j) \in \mathcal{A}+\epsilon \mathbb{B}$ where $\mathbb{B}$ is the unitary ball centered at the origin.
} 
circular path. The constant $\delta$ can be computed based on the available expression of $w_{r}$. We argue that item (iii) of Proposition 7 can always be verified by selecting $\gamma$ sufficiently small.

\section{EXPERIMENTAL VALIDATION}

We start by briefly describing the benchmark depicted in Figure 2. The robot is a Khepera ${ }^{4}$ III provided by $\mathrm{k}$-team, which is equipped with an onboard computing platform with wireless capabilities. Its coordinates are obtained by odometry. The communication is ensured via a IEEE $802.11 \mathrm{~g}$ wireless network. As a consequence, the event-triggered controller, which is located on a remote computer, verifies the transmission condition periodically every $T_{\text {eval }}=20 \mathrm{~ms}$, and not continuously ${ }^{5}$ as in Section $2-3$. This is justified by the fact that the time-interval between each position update varies from 10 to $15 \mathrm{~ms}$. Moreover, the computation time at each step is less than $1 \mathrm{~ms}$. In addition, the time for delivering a new control input is less than $5 \mathrm{~ms}$. We have verified in experiments that the value of $T_{\text {eval }}$ is sufficiently small to maintain the properties ensured by Theorem 6 . The reduction of the usage of the communication channel only concerns the transmissions from the controller to the robot, which corresponds to the dashed channel in Figure 2 (and not from the robot to the controller where periodic sampling is applied). The following parameters have been selected for the control law in (5): $c_{3}=0.7, c_{4}=0.5, c_{5}=0.8$, $\gamma=1$.

Two trajectories have been considered for which the paths are respectively an ellipse and a lemniscate. Assumptions 1 and 4 are guaranteed by using Lemma 2 . The triggering condition (12) has been implemented with $\sigma=0.5$ and we have studied the influence of $\varepsilon$. Simulations have been run over $T_{\text {simulation }}=60 \mathrm{~s}$. We have quantified the usage of the communication network with the percentage:

$$
r:=100 \times \text { number of control updates } \times \frac{T_{\text {eval }}}{T_{\text {simulation }}} .
$$

The performance of the controller is evaluated by the constant $e$ which is the average value of $\mid\left(x-x_{r}, y-y_{r}, \theta-\right.$ $\left.\theta_{r}\right) \mid$ over the second half of the experiment, i.e.

$$
e:=\frac{2}{T_{\text {simulation }}} \int_{\frac{1}{2} T_{\text {simulation }}}^{T_{\text {simulation }}}\left|\left(\begin{array}{c}
x(t)-x_{r}(s) \\
y(t)-y_{r}(s) \\
\theta(t)-\theta_{r}(s)
\end{array}\right)\right| \mathrm{d} s .
$$

In that way, $e$ quantifies the tracking accuracy of the robot after the transient period. Table 1 shows that the controller only uses the communication channel $13.59 \%$ of the time, in the worst case, and that this quantity can be significantly decreased by varying $\varepsilon$. Thus, we see that to increase $\varepsilon$ reduces the usage of the communication channel at the price of degraded tracking performances. It would be interesting to compare the event-triggered controller with a time-triggered one. Unfortunately, we are not aware of any analytical result which allows to derive an upper bound on the sampling period at which the controller (5) should be periodically emulated (this is again due to the fact that the Lyapunov function $V$ in (10) is weak). Nevertheless, we have implemented the same

\footnotetext{
4 http://www.k-team.com/

5 We actually have a periodic event-triggered controller, see Heemels et al. [2013].
}

\begin{tabular}{ccccc}
$\varepsilon$ & \multicolumn{2}{c}{ Ellipse } & \multicolumn{2}{c}{ Lemniscate } \\
\hline & $r$ & $e$ & $r$ & $e$ \\
\hline 0.01 & $6.38 \%$ & 0.0122 & $13.59 \%$ & 0.0159 \\
0.02 & $7.46 \%$ & 0.0129 & $9.51 \%$ & 0.026 \\
0.05 & $0.98 \%$ & 0.048 & $2.9 \%$ & 0.0542 \\
0.1 & $0.5 \%$ & 0.0731 & $1.37 \%$ & 0.1064 \\
0.2 & $0.74 \%$ & 0.1564 & $0.87 \%$ & 0.1947 \\
0.5 & $0.67 \%$ & 0.5256 & $0.7 \%$ & 0.6371
\end{tabular}

Table 1. Values of $r$ and $e$ for different $\varepsilon$

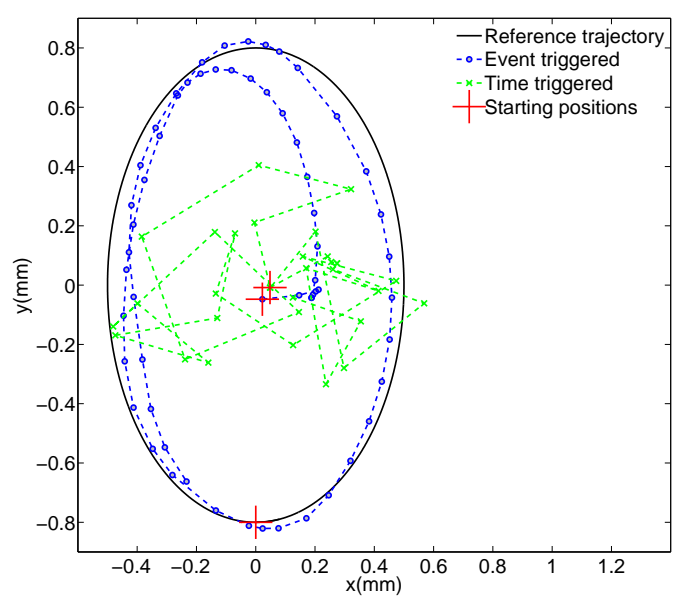

Fig. 3. Experimental results for the closed-loop trajectories when the reference describes an ellipse.

controller and we have transmitted the control input to the robot every $T_{\text {sampling }}$ seconds. For a fair comparison, we have selected $T_{\text {sampling }}$ to be the average inter-transmission interval of the corresponding event-triggered controller. We have obtained $T_{\text {sampling }}=1.904 \mathrm{~s}$ for the ellipse and $T_{\text {sampling }}=1.234 \mathrm{~s}$ for the lemniscate. In that way, both controllers do generate a similar amount of transmissions over an experiment. Figures 3 and 4 show the trajectories for each controller with $\varepsilon=0.01$ for the ellipse and the lemniscate reference trajectories respectively. Note that $\varepsilon$ impacts the average inter-transmission interval and thus the sampling period of the time-triggered controller. We see that the time-triggered controller is not able to ensure the tracking objective as opposed to the event-triggered controller. This is due to the fact that the latter adapts the frequency of control input updates when it is needed. The experiments have shown that we need to take sampling periods which are approximately four times smaller than the ones above for the time-triggered closed-loop system to ensure the tracking objective.

\section{CONCLUSION}

An event-triggered feedback law for the tracking control of a mobile robot has been presented. The problem has been modeled as an hybrid system using the formalism of Goebel et al. [2012]. The designed controller consists in the law of Jiang and Nijmeijer [1997] together with an event-triggering condition inspired by Forni et al. [2010], Anta and Tabuada [2012]. We needed to resort to the an invariance principle of Sanfelice et al. [2007] to prove that the robot practically tracks the reference trajectories. Sufficient conditions for the existence of a uniform minimum inter-transmission time have been provided. The controller has been implemented on a benchmark and we have no- 


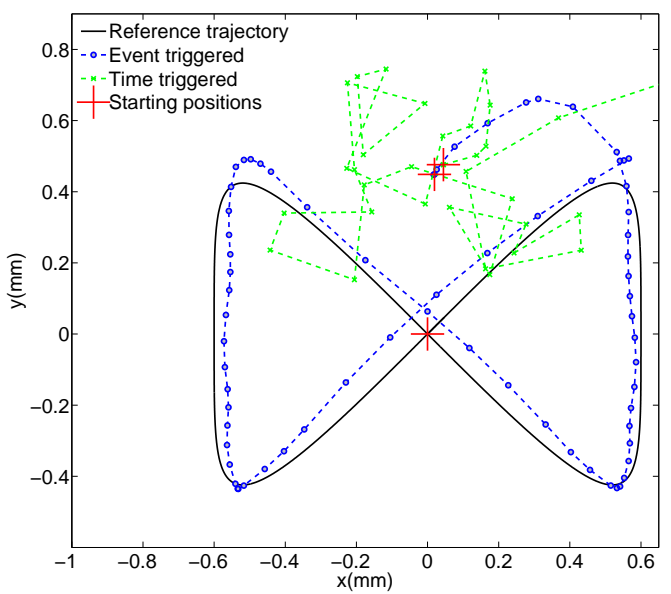

Fig. 4. Experimental results for the closed-loop trajectories when the reference describes a lemniscate.

ticed that it is able to reduce the amount of transmissions compared to periodic setups for similar performances.

\section{REFERENCES}

A. Anta and P. Tabuada. Exploiting isochrony in selftriggered control. IEEE Trans. on Automatic Control, 57(4):950-962, 2012.

K.E. Arzén. A simple event-based PID controller. In $14^{\text {th }}$ IFAC World Congress, Beijing, China, pages 423-428, 1999 .

K.J. Astrom and B.M. Bernhardsson. Comparison of Riemann and Lebesgue sampling for first order stochastic systems. In IEEE Conference on Decision and Control, Las Vegas, U.S.A., pages 2011-2016, 2002.

M.C.F. Donkers and W.P.M.H. Heemels. Output-based event-triggered control with guaranteed $\mathcal{L}_{\infty}$-gain and improved and decentralized event-triggering. IEEE Transactions on Automatic Control, 57(6):1362-1376, 2012.

F. Forni, S. Galeani, D. Nešić, and L. Zaccarian. Lazy sensors for the scheduling of measurement samples transmission in linear closed loops over networks. In IEEE Conf. on Decision and Control, Atlanta, U.S.A., pages 6469-6474, 2010.

R. Goebel, R.G. Sanfelice, and A.R. Teel. Invariance principles for switching systems via hybrid systems techniques. Syst. \& Control Letters, 57:980-986, 2008.

R. Goebel, R.G. Sanfelice, and A.R. Teel. Hybrid dynamical systems. Princeton, U.S.A., 2012.

W.P.M.H. Heemels, R.J.A. Gorter, A. van Zijl, P.P.J. van den Bosch, S. Weiland, W.H.A. Hendrix, and M.R. Vonder. Asynchronous measurement and control: a case study on motor synchronization. Control Engineering Practice, 7(12):1467-1482, 1999.

W.P.M.H. Heemels, J.H. Sandee, and P.P.J. van den Bosch. Analysis of event-driven controllers for linear systems. International Journal of Control, 81(4):571590, 2009.

W.P.M.H. Heemels, M.C.F. Donkers, and A.R. Teel. Periodic event-triggered control for linear systems. IEEE Transactions Automatic Control, 2013.

T. Henningsson and A. Cervin. Comparison of LTI and event-based control for a moving cart with quantized position measurements. In Proceedings of the European Control Conference, 2009.

T. Henningsson, E. Johannesson, and A. Cervin. Sporadic event-based control of first-order linear stochastic systems. Automatica, 44:2890-2895, 2008.

Z.-P. Jiang and H. Nijmeijer. Tracking control of mobile robots: a case study in backstepping. Automatica, 33 (7):1393-1399, 1997.

Y. Kanayama, Y. Kimura, F. Miyazaki, and T. Noguchi. A stable tracking control method for an autonomous mobile robot. In ICRA (IEEE International Conference on Robotics and Automation), pages 384-389, 1990.

D. Lehmann and J. Lunze. Extension and experimental evaluation of an event-based state-feedback approach. Control Engineering Practice, 19(2):101-112, 2011.

D. Nešić and A.R. Teel. Input-output stability properties of networked control systems. IEEE Trans. on Aut. Control, 49:1650-1667, 2004.

R. Postoyan, A. Anta, D. Nešić, and P. Tabuada. A unifying Lyapunov-based framework for the event-triggered control of nonlinear systems. In IEEE Conf. on Decision and Control and European Control Conf., Orlando, U.S.A., pages 2559-2564, 2011.

R. Postoyan, N. van de Wouw, D. Nešić, and W.P.M.H. Heemels. Emulation-based tracking solutions for nonlinear networked control systems. In IEEE Conference on Decision and Control, Hawaï, U.S.A., pages 740-745, 2012.

R.G. Sanfelice, R. Goebel, and A.R. Teel. Invariance principles for hybrid systems with connections to detectability and asymptotic stability. IEEE Transactions on Automatic Control, 52(12):2282-2297, 2007.

A. Seuret and C. Prieur. Event-triggered sampling algorithms based on a Lyapunov function. In IEEE Conference on Decision and Control and European Control Conference, Orlando, U.S.A., pages 6128-6133, 2011.

P. Tabuada. Event-triggered real-time scheduling of stabilizing control tasks. IEEE Transactions on Automatic Control, 52(9):1680-1685, 2007.

P. Tallapragada and N. Chopra. On event triggered trajectory tracking for control affine nonlinear systems. In IEEE Conference on Decision and Control and European Control Conference, Orlando, U.S.A., Orlando, U.S.A., pages 5377-5382, 2011.

J.J. Téllez-Guzmán, F. Guerrero Castellanos, S. Durand, N. Marchand, and R. Lucio-Maya. Event-based LQR control for attitude stabilization of a quadrotor. In Proc. of the IFAC Latinamerican Control Conf., 2012. 